\title{
Kernel Based Symmetry Measure
}

\author{
Bertrand Zavidovique $^{2}$ and Vito Di Gesù ${ }^{1}$ \\ 1 IEF, University of Paris XI, ORSAY, France \\ ${ }^{2}$ DMA, Università di Palermo, Italy
}

\begin{abstract}
In this paper we concentrate on a measure of symmetry. Given a transform $S$, the kernel $S K$ of a pattern is defined as the maximal included symmetric sub-set of this pattern. A first algorithm is outlined to exhibit this kernel. The maximum being taken over all directions, the problem arises to know which center to use. Then the optimal direction triggers the shift problem too. As for the measure we propose to compute a modified difference between respective surfaces of a pattern and its kernel. A series of experiments supports actual algorithm validation.
\end{abstract}

\section{Introduction}

This paper deals with capturing approximate symmetry from pictures, wherever it could show in them.

Symmetry is a prevalent perceptive feature for humans. From the survey by Zabrodsky [ZA1], we retain results corroborating our own findings for machines: a) saliency of vertical symmetry associated with a mental rotation: detected symmetry is in the order vertical, horizontal, bent and then rotational; b) symmetry of parts near the axis contribute more than symmetry of further parts near edges, themselves more critical than regions in between.

Symmetry is important in machine vision too as proven by an extensive literature. See OM1 for a recent quite interesting survey. Models of symmetry suffer three major drawbacks: $\mathbf{d}_{\mathbf{1}}$ edges mainly support symmetry detection; $\mathbf{d}_{\mathbf{2}}$ perfect symmetry is targeted; $\mathbf{d}_{\mathbf{3}}$ the center of mass is assumed to be the focus of attention.

Similar difficulties were long solved for other features as edges, regions or motion in actually measuring the phenomenon - edginess, uniformity, set-direction - to make decisions from the measure rather than from a strict distance. We addressed $\mathbf{d}_{\mathbf{1}}$ in a previous work DG3] leading to define iterative transforms as the IOT that better accounts for the inner object. In the present paper we tackle the three $\mathbf{d}_{\mathbf{i}}$-difficulties together. After a short bibliography in section 2 reviewing elementary tools, we first introduce the notion of a kernel in section 3. It stems logically from IOT through a gauge classical in functional analysis : maximal included (resp. minimal including) set with same property. In section 4 , a series of experiments on both binary and grey scaled pictures allow to check proposed techniques, their sensitivity to the center position and the validity of the degree of symmetry. Discussion and further comments conclude the paper. 


\section{State of the Art}

The Symmetry Axial Transform (SAT) BL1] can be considered seminal for symmetry detection starting from borders of an object. Exhibiting centers of maximal circles, $S A T$ retrieves only axes of symmetry already included in the medial axis. Some limitations are solved by the Smoothed Local Symmetry BR1]. Global symmetry, if any, is retrieved from the local curvature of contours, through the locus of mid-edge-point pairs. Likewise, Sewisy SE1 couples the Hough transform with geometric symmetry to exhibit candidate ellipse centers. In [FU1] polygonal approximations of contours are broken into elements (e.g. Delaunay/Voronoi method) of which axial features are pieced together. Gray level symmetry was firstly investigated in [MA1], where the descriptor is based on cross correlation. In [DG1], symmetries stem from evaluating the axial moment of a body around its center of gravity. This descriptor has been applied at a local level to define the Discrete Symmetry Transform (DST). In [MN1, local reflectional symmetry is computed in convolving with the first and second derivative of Gaussian's. Both a "measure" of symmetry and an axis orientation are provided at each point. Shen [SH1] or DuBuff BI] use complex moments associated with Fourier or Gabor transforms for image approximation.

In [KI1, authors introduce several descriptors from Marola's one, further extended to finite supports and varying scales based on Radon and Fourier transforms. A global optimization approach is implemented by a probabilistic genetic algorithm to speedup computations. Along the same line, Shen and al. SH1 detect symmetry in seeking out the lack of it. The asymmetric part of their measure (energy) is null for a set of pixels invariant through horizontal reflection, hence the minimization. In [CR1] (see also DG2]), a vector potential is similarly constructed from the gradient field. Edge and symmetry lines are extracted through a topographical analysis of the vector field (i.e. curl of the vector potential) at various scales. Symmetry axes are lines where the curl of the vector vanishes and edges are where the divergence of the potential vanishes. Yeshurun and al. RE1 build on the Blum-Asada vein: they quantify a potential for every pixel to be center of symmetry, based on pairs of edge points tentatively symmetric from their gradient vectors. A symmetry degree is assigned to every pair within a pixel neighborhood and a weighted combination of these makes the potential, whose local maxima provide a measure depending on both intensity and shape.

Some methods provide symmetry descriptors for measures to be computed, others aim at straight symmetry measures. The difference is obvious in comparing for instance Cross's and Yeshurun's. Finally, preceding works show that: 1- introducing true measures helps building more abstract versions of distances, more suitable for approximate comparison of objects; 2- sets which measures apply on may be "sets of pixels or vectors" (shapes) or "sets of patterns" (class transforms): in either case set operations, as Minkowski's ones, are worth considered. They do not limit to contours and bridge logic with geometry.

Before to explain why and how we put these ingredients together, let us conclude by pointing out two more works that fit the algorithmic line above and are 
the closest to ours. It makes clear the main contributions of this paper respective to previous work. R. Owens OM2 searches explicitly for a measure to indicate approximate bilateral symmetry of an object. But she defines tentative symmetries from the principal axes of inertia, whence the centroid again. Although it is not mentioned, her measure based on the sum of absolute differences of grey levels in symmetric pairs amounts to a slightly modified $\mathrm{L}_{1}$-difference between the object and a maximal-for-inclusion symmetric version of it in the given direction. Kazhdan et al. KA1] use explicitly the same idea of a difference $\left(\mathrm{L}_{2}\right.$ in their case) between the image and its closest symmetric version. They need a measure that integrates all reflective invariance about a bundle of straight lines (or planes in 3-D) and a center is necessary to this representation.

\section{The New Symmetry Measure}

In previous papers DG3 we defined the IOT that is a map product of iterated morphological erosion and symmetry detection.

Definition 1. The Symmetry Transform, S, on a continuous object $X \subset R^{2}$ is:

$$
S_{\alpha}(\mathrm{X})=\int_{X} m(x) \times \rho^{2}(x, r(\alpha)) d x \quad \text { for } \quad \alpha \in[0, \pi[
$$

where, $r(\alpha)$ is the straight line with slope $\alpha$ passing through the center of gravity of the object $X, m(x)$ is the mass of the object in $x \in X$, and $\rho$ is a distance function of $x$ from the straight line. $\diamond$

Each value of $S$ represents the axial moment of $X$.

Definition 2. The Iterated Object Transform, IOT, is given by:

$$
\operatorname{IOT}_{\alpha, 1}(X)=S_{\alpha}(X) \quad ; \quad \operatorname{IOT}_{\alpha, n}(X)=S_{\alpha}\left[(\mathrm{E})^{n-1}(X)\right] \quad \text { for } n>1
$$

$(E)^{n}$ stands for the morphological erosion by the unit sphere (or any other suitable structuring element would any suitable a priori information be available), iterated $n$ times.

The number of iterations depends on the size of the input image and on the distribution of the gray levels. The $S$ transform is thus computed on progressively shrunk versions of the binary input image or on steadily intensity reduced versions of the gray level input image, until some predefined decrease or a minimum of intensity is reached.

The iterated elongation, $\eta_{n}(X)$, is defined as follows:

$$
\eta_{n}(X)=\frac{\min _{\alpha \in[0, \pi[}\left\{\operatorname{IOT}_{\alpha, n}(X)\right\}}{\max _{\alpha \in[0, \pi[}\left\{\operatorname{IOT}_{\alpha, n}(X)\right\}}
$$

It represents dynamic changes of $X$ shapes indicators versus $n$. Since in most cases, $\eta$ curves become flat or show some other type of constancy after a certain number of erosions, it was conjectured that any pattern larger than the 
structuring element would have a symmetric kernel that IOT reveals: indeed, in eroding a pattern at least one pixel remains to meet the definition. Let us call IOTK this pattern. The intuitive idea here is that the closer the kernel to the pattern, the more symmetric pattern. Unfortunately it is easy to design examples (see Figure 1) where the IOTK is as "far" as one wants from the pattern. Never the less, such a symmetric pattern, bound to the more or less symmetric object under study, should then contribute to a symmetry measure, and more generally to a feeling (sensing) of symmetry by machines.

Remark 1: when it proves necessary, this included version of the kernel could be balanced by the including version obtained by dilation.

Following commonly used gauges in functional analysis, a possible first answer with a flavor of optimality would be maximal included symmetric pattern resp. minimal including symmetric pattern : extremal then subjects to the measure.

Definition 3. The $S$-kernel of the pattern $P-S K(P)$ - is the maximal for inclusion symmetric (pattern) subset of $P$.

A first algorithm to be discussed and optimized is to compute a symmetric pattern included in the given one, ptrn, starting from an initial center, $G$, and iterate the process for all $G$ 's and all directions (Figure 1) until the maximum is reached. Here is the main core loop:

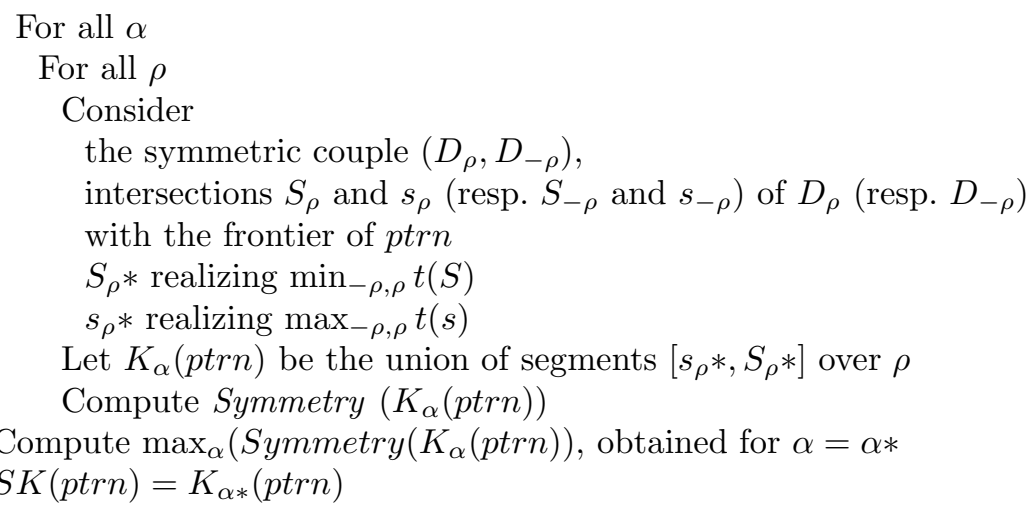

The meaning of $S_{\rho}$ and $s_{\rho}$ (resp. $S_{-\rho}, s_{-\rho}$ ) and $D_{\rho}$ (resp. $D_{-\rho}$ ) is illustrated in Figure1a. In case of more than 2 intersections (concavities) the algorithm extends by segments in a natural way. Actually the algorithm implementation makes use of the $\min$ (resp. $\max$ ) operators pixel to pixel in $D_{\rho}$ and $D_{-} \rho$ respectively, to exhibit the kernel without any prior thresholding (see Figures $3 \mathrm{c}$ and $\mathrm{d}$ and $4 \mathrm{~b}$ and d) for result samples). Except for some pathological patterns and following Definition 1, the center of mass is conjectured a good enough approximation to balance the pattern from. Therefore, not to span the all search space, $G$ is set first to the center of mass of ptrn so that the initial state be likely close to the global optimum. The result for the pattern in Figure 19 is the grey 


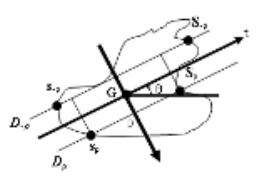

(a)

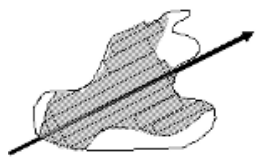

(b)

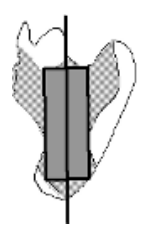

(c)

Fig. 1. (a) Sketch of the kernel detection algorithm; (b) the kernel of the pattern in (a); (c) expanding the IOTK of the pattern in (a) into the kernel

shaded part as given in Figure 1 b. One has then to relate the latter kernel to the former indicators $($ IOTK $)$. Let us assume we applied the IOT and found a stable pattern after multiple erosion, like the dark rectangle in the Figure 1; (meaning after that, erosion will confirm symmetry and $\eta$ remains constant). Starting from there we expand the pattern and mark progressively every where the expansion intersects the border, together with the symmetric pixel wrt. the axis. Every marked pixel is not expanded further. That way the kernel should be obtained again, provided the center of mass be stable. The preceding algorithm is a line by line version of the one here, and that makes the expected link.

Remark 2: the center of mass varies from the kernel to the pattern, still all definitions so far assume that tentative symmetry axes pass by this center.

This introduces an additional question: how to define the likely symmetry axis where to compute the kernel from? For instance, let be $\mu=\operatorname{argMaxSymmetry}$ $(p t r n)$. How does $K_{\mu}(p t r n)$ compare with $K(p t r n)$ ? How do their respective Symmetry relate? In most cases $K_{\mu}($ ptrn $)$ should be a good enough gauge of $K(p t r n)$, or their difference will be most indicative. The last part of experiments is devoted to answering the question, in checking results over translations of the axis.

In order to test the proposed algorithm we compute a measure of symmetry classically defined as:

$$
\lambda=1-\frac{\operatorname{Area}(D)}{\operatorname{Area}(A)}
$$

with $A$, the pattern, $B$, its kernel, and $\operatorname{Area}(D)=\operatorname{Area}(A-B)$

It remains a robust first approximation where $\lambda=1$ if $\operatorname{Area}(B)=\operatorname{Area}(A)$. In any case, different ways of limiting the pattern should be compared to the one here, that is based on the sole erosion.

\section{Experimental Results and Further Comments}

In this section we show some results of the S-kernel algorithm applied to synthetic and real images. The purpose of experiments can be summarized as: 


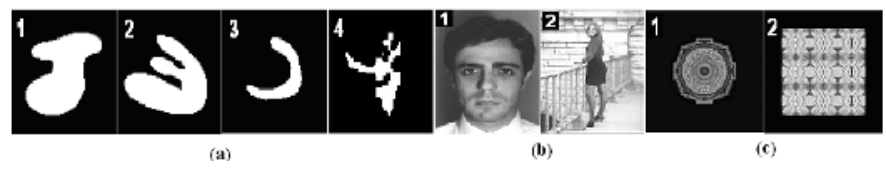

Fig. 2. Sample of images used for experiments: (a) binary; (b) gray level; (c) textured

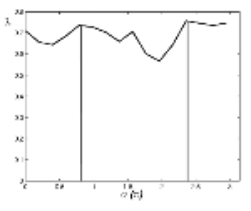

(a)

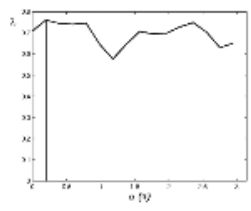

(b)

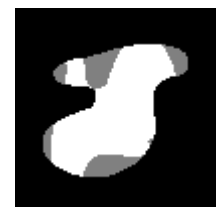

(c)

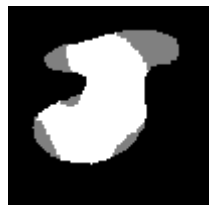

(d)

Fig. 3. Finding the direction of maximal symmetry for the binary pattern in Figure 2 1 through $G$ (a) and through shifted centers around $G(\mathrm{~b})$ and actual $S K$ superimposed to the input pattern - in white - according to $G$ (c) and the shifted center (d)

1 check the sensitivity of the method to the center position. Two cases will be compared: a) through $G$; b) shift from $G$;

2 validate the method ability to measure a degree of symmetry, by comparing $|\eta(S K(p t r n))-\eta(p t r n)|$ with $\lambda$, and $\eta(I O T K)$ with $\eta(S K(p t r n))$;

All experiments are parameterized by direction and completed on both binary and gray level images (see Figure 2).

First, we consider the variation of $\lambda$ with the angle $\alpha$ used to compute $S K(p t r n)$ on the binary pattern in Figure 2a-1. Figure 3 a shows $\lambda$ versus $\alpha$ when the pole is $G$; in this example the maximum similarity is $\lambda=0.76$ and the direction is $\alpha=135^{\circ}$. Figure 3b shows $\lambda_{M}(\alpha)=\max \{\lambda(\alpha, C), \quad G-S \leq C \leq G+S\}$ when the pole is shifted around $G$ for all $\alpha^{\prime}$ 's; the maximum similarity is $\lambda=0.76$ and the direction is $\alpha=11.25^{\circ}$. The human perception, bound to display, would favor the local minimum near $\pi / 2$. The variation of $\lambda$ for $\alpha=\pi / 2$ versus the shift of $\mathrm{C}$ shows maximum similarity for a negative five pixels shift from $G$. White pixels in Figure 3r,d display kernels obtained when $\lambda_{\max }=\max _{\alpha}\{\lambda\}$ for fixed and varying centers respectively. We tested the robustness of the algorithms in rotating the images by $45^{\circ}$; it comes $\lambda_{G}=0.76, \alpha_{G}=45.00^{\circ}$ and $\lambda_{C}=0.78, \alpha_{C}=45.00^{\circ}$ for image $1 \mathrm{~b}, \lambda_{G}=0.92, \alpha_{G}=135.00^{\circ}$ and $\lambda_{C}=0.95$, $\alpha_{C}=45.00^{\circ}$ for image $2 \mathrm{~b}$. Table 1 reports the results for all Figure 2 subscripts $G$ and $C$ indicate respective processing through $G$ and with shifting. The two rightmost columns display the maximum of the Object Symmetry Transform, $O S T_{\max }=\max _{0 \leq \alpha<\pi}\{O S T(\alpha)\}$, and corresponding direction, $\alpha_{O S T}$, obtained with the algorithm in DG3. Results are comparable, but the kernel algorithm is more accurate with directions. Figures 4 a,b show plots of $\lambda_{G}$ and $\lambda_{C}$ for the image $1 c$. The ability of the proposed kernel operators is evident in detecting global object symmetries. In fact, the mean values and the variance of $\lambda_{G}$ and 
Table 1. Comparing kernel results and $O S T$ on images in Figure 2

\begin{tabular}{ccccccc}
\hline Image & $\lambda_{G}$ & $\alpha_{G}$ & $\lambda_{C}$ & $\alpha_{C}$ & $O S T$ & $\alpha_{O S T}$ \\
\hline 1a & 0.76 & $135.00^{\circ}$ & 0.76 & $11.25^{\circ}$ & 0.86 & $112.50^{\circ}$ \\
2a & 0.74 & $90.00^{\circ}$ & 0.79 & $33.75^{\circ}$ & 0.93 & $101.00^{\circ}$ \\
3a & 0.82 & $157.50^{\circ}$ & 0.76 & $22.50^{\circ}$ & 0.87 & $56.25^{\circ}$ \\
4a & 0.76 & $0.00^{\circ}$ & 0.80 & $0.00^{\circ}$ & 0.80 & $0.00^{\circ}$ \\
1b & 0.80 & $90.00^{\circ}$ & 0.80 & $90.00^{\circ}$ & 0.72 & $90.00^{\circ}$ \\
2b & 0.70 & $90.00^{\circ}$ & 0.89 & $90.00^{\circ}$ & 0.92 & $45.00^{\circ}$ \\
1c & 0.99 & $90.00^{\circ}$ & 0.99 & $135.00^{\circ}$ & 0.90 & $90.00^{\circ}$ \\
2c & 0.99 & $0.00^{\circ}$ & 0.99 & $90.00^{\circ}$ & 0.96 & $0.00^{\circ}$ \\
\hline
\end{tabular}

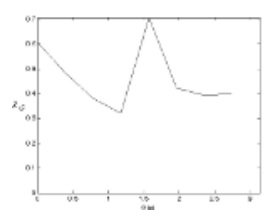

(a)

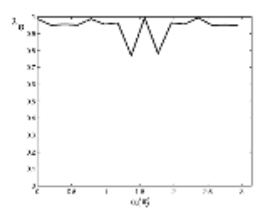

(e)

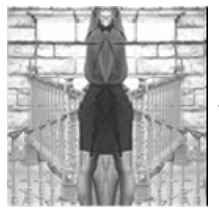

(b)

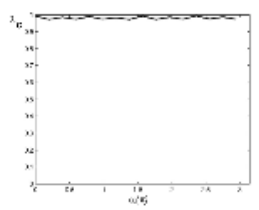

(f)

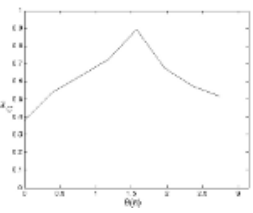

(c)

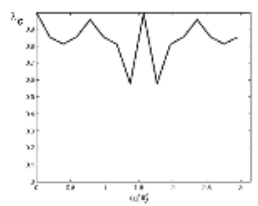

(g)

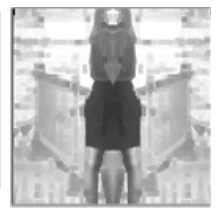

(d)

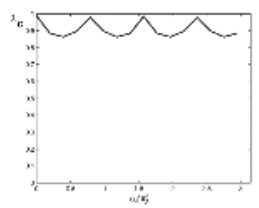

(h)

Fig. 4. Plot of $\lambda_{G}$ and $\lambda_{C}$ for the image $2 b$ (resp. a and c) with corresponding kernels (resp. b and d), and for the image 1c (resp. e and f) and 2c (resp. g and h) of Figure 2

$\lambda_{C}$ are $(0.94,0.2)$ and $(0.94,0.01)$ respectively indicating the circular symmetry of the image 1c. Note that $\lambda_{C}$ is a more robust indicator of the circularity. Same considerations hold for the image 2c (see Figure 4). For comparison, the mean value and variance of the $O S T$ of image $1 c$ are $(0.89,0.01)$ confirming the circular symmetry too.

\section{Concluding Remarks}

This paper describes a new measure of axial symmetry derived from an object feature named "symmetry-kernel". The symmetry kernel of an object is the maximal subpart that is symmetric regarding a given direction. An algorithm is proposed, based on the computation of the $O S T$ for bilateral symmetry. It is rotation invariant and provides quite interesting results. However, it is computationally expensive on conventional computers as it computes line intersections and distances. Eventually, it is sensitive to numerical precision. It was tested on 
both synthetic and real data. Experiments show the ability of the symmetrykernel to detect the main directionality of objects. The distance between an object and its kernel is a crucial point needing further investigation.

\section{References}

[CE1] Clarke, F., Ekeland, I.: Nonlinear oscillations and boundary-value problems for Hamiltonian systems. Arch. Rat. Mech. Anal. 78 (1982) 315-333

[BL1] Blum,H., Nagel, R.N.: Shape description using weighted symmetric axis features. Pattern recognition, 10, (1978) 167-180.

[BR1] Brady M., Asada H.: Smoothed Local Symmetries and their implementation. The Int.J. of Robotics Research, 3(3), (1984) 36-61.

[CR1] Cross, A.D.J., Hancock, E.R.: Scale space vector fields for symmetry detection. Image and Vision Computing, 17(5-6), (1999) 337-345.

[DG1] Di Gesù, V., Valenti, C.: Symmetry operators in computer vision. Vistas in Astronomy, Pergamon, 40(4), (1996) 461-468.

[DG2] Di Gesù, V. Valenti, C.: Detection of regions of interest via the Pyramid Discrete Symmetry Transform. Advances in Computer Vision (Solina, Kropatsch, Klette and Bajcsy Eds.), Springer-Verlag, (1997).

[DG3] DiGesù, V., Zavidovique, B.: A note on the Iterative Object Symmetry Transform. Pattern Recognition Letters, 25, (2004) 1533-1545.

[KA1] Kazhdan, M., Chazelle, B., Dobkin D., Finkelstein, A., Funkhouser, T.: A reflective symmetry descriptor. $7^{\text {th }} E C C V$, (2002) 642-656.

[KI1] Kiryati, N., Gofman, Y.: Detecting symmetry in grey level images (the global optimization approach). preprint, (1997).

[MA1] Marola, G.: On the detection of the axes of symmetry of symmetric and almost symmetric planar images. IEEE Trans.of PAMI, 11, (1989) 104-108.

[MN1] Manmatha, R., Sawhney, H.: Finding symmetry in Intensity Images", Tech.Rep., (1997).

[OM1] O'Mara, D.: Automated facial metrology, chapter 4: Symmetry detection and measurement. PhD thesis, Feb. (2002).

[OM2] O'Mara, D., Owens, R.: Measuring bilateral symmetry in digital images. IEEE TENCON, Digital signal processing applications, (1996).

[SE1] Sewisy, A., Lebert F.: Detection of ellipses by finding lines of symmetry inthe images via an Hough transform applied to staright lines. Image and Vision Computing, 19(12), (2001) 857-866.

[SH1] Shen, D., Ip, H., Teoh, E.K.: An energy of assymmetry for accurate detection of global reflexion axes. Image Vision and Computing, 19, (2001) 283-297.

[FU1] Fukushima, S.: Division-based analysis of symmetry and its applications. IEEE PAMI, 19(2), (1997).

[SH1] Shen, D., Ip, H., Cheung, K.T., Teoh, E.K.:Symmetry detection by Generalized complex moments: a close-form solution. IEEE PAMI, 21(5), (1999).

[BI1] Bigun, J., DuBuf, J.M.H.: N-folded symmetries by complex moments in Gabor space and their application to unsupervized texture segmentation. IEEE PAMI, 16(1), (1994).

[RE1] Reisfeld, D., Wolfson, H., Yeshurun, Y.:Detection of interest points using symmetry. 3rd IEEE ICCV Osaka, (1990).

[ZA1] Zabrodsky, H.: Symmetry - A review. Tech.Rep.90-16, CS Dep. The Hebrew Univ.Jerusalem, 1990. 